\title{
Serum biomarker for diagnostic evaluation of pulmonary arterial hypertension in systemic sclerosis
}

\author{
Lisa M. Rice ${ }^{1 *}$, Julio C. Mantero ${ }^{1}$, Eric A. Stratton', Rod Warburton², Kari Roberts ${ }^{2}$, Nicholas Hill ${ }^{2}$, Robert W. Simms ${ }^{1}$, \\ Robyn Domsic ${ }^{3}$, Harrison W. Farber ${ }^{1}$ and Robert Layfatis ${ }^{3}$
}

\begin{abstract}
Background: Systemic sclerosis-associated pulmonary arterial hypertension (SSc-PAH) is one of the leading causes of death in SSc. Identification of a serum-based proteomic diagnostic biomarker for SSc-PAH would allow for rapid noninvasive screening and could positively impact patient survival. Identification and validation of novel proteins could potentially facilitate the identification of SSc-PAH, and might also point to important protein mediators in pathogenesis.

Methods: Thirteen treatment-naïve SSC-PAH patients had serum collected at time of diagnosis and were used as the discovery cohort for the protein-expression biomarker. Two proteins, Midkine and Follistatin-like 3 (FSTL3) were then validated by enzyme-linked immunosorbent assays. Midkine and FSTL3 were tested in combination to identify SSc-PAH and were validated in two independent cohorts of SSC-PAH $(n=23, n=11)$.

Results: Eighty-two proteins were found to be differentially regulated in SSC-PAH sera. Two proteins (Midkine and FSTL3) were also shown to be elevated in publicly available data and their expression was evaluated in independent cohorts. In the validation cohorts, the combination of Midkine and FSTL3 had an area under the receiver operating characteristic curve (AUC) of 0.85 and 0.92 with respective corresponding measures of sensitivity of $76 \%$ and $91 \%$, and specificity measures of $76 \%$ and $80 \%$.

Conclusions: These findings indicate that there is a clear delineation between overall protein expression in sera from SSC patients and those with SSc-PAH. The combination of Midkine and FSTL3 can serve as an SSC-PAH biomarker and are potential drug targets for this rare disease population.
\end{abstract}

Keywords: Classification, Proteomic, Scleroderma, Biomarkers, Pulmonary arterial hypertension

\section{Background}

Cardiopulmonary involvement is the most common cause of morbidity and mortality in patients with systemic sclerosis (SSc). Twelve percent of SSc patients will develop SSc-associated pulmonary arterial hypertension (SSc-PAH); these patients have an estimated 50\% 3-year survival [1]. Early and accurate diagnosis of PAH is clinically challenging and relies on right heart catheterization (RHC). RHC is invasive, is not suitable for screening, and so is typically performed only on patients with a high index of suspicion

\footnotetext{
* Correspondence: lisarice@bu.edu

${ }^{1}$ Boston University School of Medicine, E5 Arthritis Center, 72 E Concord

Street, Boston, MA 0211, USA

Full list of author information is available at the end of the article
}

based on echocardiogram and other criteria that are neither highly sensitive nor specific [2-4].

Earlier diagnosis and treatment with $\mathrm{PAH}$-specific therapeutics is associated with better outcomes in patients with SSc-PAH $[5,6]$. Thus, identification of a diagnostic biomarker that would allow for rapid non-invasive screening could positively impact patient survival, supplement current screening methods, and potentially reduce the need for diagnostic RHC.

Transthoracic echocardiography is the most widely accepted screening tool for the diagnosis of SSc-PAH. Echocardiographically estimated systolic PAP (sPAP) correlates reasonably $(r=0.83)$ with RHC [7], though it has potential limitations including an $\sim 45 \%$ false positive rate [8-10]. Echocardiogram has also been studied

(C) The Author(s). 2018 Open Access This article is distributed under the terms of the Creative Commons Attribution 4.0 International License (http://creativecommons.org/licenses/by/4.0/), which permits unrestricted use, distribution, and 
in combination with other measures. PAH biomarkers such as brain natriuretic peptide (BNP) and the $\mathrm{N}$-terminal fragment of pro-BNP (NT-proBNP) have similar diagnostic accuracy as the echocardiogram [11]. Natriuretic peptide levels of have been shown to correlate with hemodynamic measurements, predict disease prognosis and mortality among those with PAH [12]. They are also generally specific for SSc-PAH, but lack sensitivity [13]. As such, other potential biomarkers, such as osteopontin, pentraxin 3, C-reactive protein (CRP), interleukin (IL)1, IL8 and tumor necrosis factor alpha (TNF $\alpha$ ) have been investigated as diagnostic tools. In preliminary studies, some have appeared superior to both BNP and NT-proBNP [14-17]. However, these findings are tempered by the fact that they are single studies and have yet to be validated.

The clinical use of gene expression for classifying different biological disease states is well established [18]. We have previously described SSc-PAH associated gene-expression patterns in peripheral blood mononuclear cells (PBMCs) that highlight IFN-regulation and monocyte/macrophage activation in disease pathogenesis $[17,19]$. Thus, we hypothesized that wide-scale proteomic studies of serum might identify a diagnostic biomarker of SSc-PAH. This hypothesis was further supported by our previous small proteomic study, in which we identified 17 cytokines associated with SSc-PAH [17].

In the present study, we utilized a proteomic technology to identify novel proteins specific to the diagnosis of SSc-PAH. We show that in combination, Follistatin-like 3 (FSTL3) and Midkine (MDK) are highly diagnostic for SSc-PAH, and have validated these biomarkers in two independent cohorts. These results suggest that serum assays in patients with PAH could be dramatically improved with identification of these serum biomarkers and, thus, aid in the earlier diagnosis of SSc-PAH.

\section{Methods}

\section{Patient selection}

Institutional review boards at Boston University, University of Pittsburgh, and Tufts University approved the collection of all serum samples and clinical data. Written informed consent was obtained from all patients before sample collection. Diagnosis of PAH was made by RHC: mean pulmonary artery pressure (mPAP) of $\geq 25 \mathrm{mmHg}$, a pulmonary capillary wedge pressure of $\leq 15 \mathrm{mmHg}$, and a pulmonary vascular resistance of $\geq 3$ Wood units [20]. Diagnosis of SSc was made by a rheumatologist and date of disease onset is recorded as date of first non-Raynaud's symptom. There are two subcategories of SSc, limited cutaneous systemic sclerosis (lcSSc) and diffuse cutaneous systemic sclerosis (dcSSc). This distinction is based on extent and location of skin disease. The majority of patients included in this study were lcSSc patients with limited skin disease, any exceptions are noted in the detailed description of each patient cohort included. All samples in the discovery cohort $(n=13)$ were collected at Boston University. Patients had limited skin disease (lcSSc) and were PAH treatment-naïve. Sera were collected at time of diagnostic RHC and were analyzed by SOMAscan (SomaLogic Boulder, CO, USA), which allowed for the capability to examine 1129 proteins simultaneously. Sixteen subjects with lcSSc and no clinical evidence of PAH (medical records were reviewed by a clinical expert in the field, forced vital capacity $[\mathrm{FVC}]>70 \%$, negative $\mathrm{RHC}$, or no record of an abnormal finding on an echocardiograph) or interstitial lung disease (ILD), diagnosed by high-resolution CT scans (HRCTs), served as controls. Protein data were analyzed for differences in lcSSc-PAH patients (Wilcoxon signed-rank test). Analytes for further study were selected based on the q values (false discovery rates $[F D R]<0.1)$. These parsed analytes were clustered and visualized using the $R$ environment for statistical computing (version 3.2.1). The clustering was unsupervised and used Spearman's correlation and average linkage. Identification of pathways linked to the differentially expressed proteins was performed using Qiagen's Ingenuity Pathway Analysis (IPA, Qiagen, Redwood City, CA, USA). Subjects in validation cohorts diagnosed with ILD based on HRCTs were included when measured FVC was $>70 \%$. The discovery cohort was from Boston University, and the validation cohort one was from both Tufts University as well as Boston University and validation cohort two was from University of Pittsburgh.

Subjects enrolled with signed informed written consent by the institutional review board of Boston University also included patients with dcSSc, as well as lcSSc subjects showing evidence of ILD (confirmed by HRCT) and healthy controls. Sera from these subjects were also analyzed by SOMAScan, after collection into a serum separator tube, allowed to clot, aliquoted, and stored at $-80{ }^{\circ} \mathrm{C}$.

\section{ELISA validations and statistical analysis}

Concentrations of candidate proteins were measured in duplicate using commercial ELISA kits for FSTL3 (DFLRG0: R\&D Systems, Minneapolis, MN, USA), or for MDK (ab193761: Abcam, Cambridge, MA, USA).

Putative biomarker protein concentrations were $\log 2$-transformed to better approximate a normal distribution and obtain reliable odds ratio (OR) estimates [21]. Wilcoxon signed-rank test were used to test the difference in SSc analyte concentrations from lcSSc-PAH. Models of discreet variables were 
developed using logistic regression and multiple logistic regression. Sensitivity and specificity of discrete variables were assessed using receiver operating characteristic (ROC) curves, plotting the sensitivity on the $\mathrm{y}$-axis and 1-specificity on the $\mathrm{x}$-axis. The area under the curve (AUC) of a ROC was computed to provide a global measure of performance and for comparing biomarker performance, with AUC of 0.90-.1 considered excellent, $0.80-0.90$ considered good, $0.7-0.8$ considered fair, $0.6-0.7$ considered poor, $0.5-0.06$ considered without value. Youden's J statistic was used to calculate the best threshold level of the biomarker that gives equal weight to sensitivity and specificity [22].

Publicly available gene expression data were downloaded from GEO: http://www.ncbi.nlm.nih.gov/geo/. The data included five data sets with relevant SSc subpopulations. The first data set was derived from the peripheral blood mononuclear cells (PBMCs) of lcSSc subjects with $(n=15)$ and without $(n=21)$ PAH. The data set was downloaded from GEO accession number GSE19617 [17]. A second data set from the PBMCs of PAH subjects including a systemic sclerosis subset. The data was downloaded from GEO accession number GSE33463 [23]. lcSSc subjects were with $(n=37)$ and without $(n=19) \mathrm{PAH}$. The third data set was derived from PBMCs of patients with SSc-PAH $(n=10)$ and SSc without $(n=10)$. The data set was downloaded with the GEO accession number GSE22356 [24]. The second type of data was derived from lung tissue from transplant subjects, there were $n=6$ Ssc subjects with PAH and $n=9$ subjects with normal lungs. The data set was downloaded with the GEO accession number GSE48149 [25]. The third type of data was derived from skin tissue of SSc subjects $(n=61)$ and healthy controls $(n=36)$. The data set

Table 1 Clinical characteristics

\begin{tabular}{|c|c|c|c|c|c|c|}
\hline \multirow[t]{2}{*}{ Baseline demographics } & \multicolumn{2}{|c|}{ Discovery cohort } & \multicolumn{2}{|c|}{ Validation cohort 1} & \multicolumn{2}{|c|}{ Validation cohort 2} \\
\hline & ICSSC-PAH & IcSSc-no PAH & ICSSC-PAH & IcSSc-no PAH & ICSSC-PAH & ICSSc-no PAH \\
\hline Age (year) & $n=13$ & $n=16$ & $n=23$ & $n=12$ & $n=11$ & $n=18$ \\
\hline Mean (SD) & $65(7.4)$ & $50(14)$ & $66(8.6)$ & $54(17.8)$ & $66(9.4)$ & $62(8.5)$ \\
\hline Median (range) & $65(56-81)$ & $57(25-70)$ & $64(52-85)$ & $52(26-76)$ & $66(52-81)$ & $63(46-79)$ \\
\hline \multicolumn{7}{|l|}{ Sex } \\
\hline Female, \% (n) & $92 \%(12)$ & $87.5 \%(14)$ & $83 \%(19)$ & $92 \%(11)$ & $100 \%(11)$ & $83 \%(15)$ \\
\hline Male,\% (n) & $8 \%(1)$ & $12.5 \%(2)$ & $17 \%(4)$ & $8 \%(1)$ & $0 \%(0)$ & $17 \%(3)$ \\
\hline \multicolumn{7}{|l|}{ mPAP (mmHg) } \\
\hline Mean (SD) & $46(9.1)$ & - & $42(12.3)$ & - & $40(10.8)$ & - \\
\hline Median (range) & $45(34-68)$ & - & $43(26-69)$ & - & $42(25-54)$ & - \\
\hline \multicolumn{7}{|l|}{ mPCWP (mmHg) } \\
\hline Mean (SD) & $10(2.7)$ & - & $10(4.3)$ & - & $10(3.2)$ & - \\
\hline Median (range) & $11(4-14)$ & - & $11(1-15)$ & - & $9(5-15)$ & - \\
\hline \multicolumn{7}{|l|}{ PVR (Woods units) } \\
\hline Mean (SD) & $9(4.9)$ & - & $8(5.3)$ & - & $8(4.1)^{a}$ & - \\
\hline Median (range) & $8(5-24)$ & - & $6(3-27)$ & - & $7(3-13)^{a}$ & - \\
\hline \multicolumn{7}{|l|}{ ILD (Dx by HRCT) } \\
\hline Positive, \% (n) & $7 \%(1)$ & - & $13 \%(3)$ & - & $0 \%(0)$ & - \\
\hline Negative, \% (n) & $83 \%(12)$ & - & $87 \%(20)$ & - & $100 \%(11)$ & - \\
\hline \multicolumn{7}{|l|}{ Treatment } \\
\hline Treated, \% (n) & $0 \%(0)$ & - & $48 \%(11)$ & $\approx$ & $100 \%(11)$ & - \\
\hline Untreated, \% (n) & $100 \%(13)$ & - & $52 \%(12)$ & - & $0 \%(0)$ & - \\
\hline \multicolumn{7}{|l|}{ BNP (pg/mL) } \\
\hline Mean (SD) & $261(313)$ & - & $411(494)$ & - & - & - \\
\hline Median (range) & $140(42-1054)$ & - & $148(12-1630)$ & - & - & - \\
\hline \multicolumn{7}{|l|}{ Date of sample } \\
\hline At time of RHC, \% (n) & 100\% (13) & - & $91 \%(21)$ & - & $36 \%(4)$ & - \\
\hline
\end{tabular}

${ }^{a}$ Missing values

BNP, brain natriuretic peptide; IcSSc-PAH, limited cutaneous systemic sclerosis pulmonary arterial hypertension; ILD, interstitial lung disease; mPAP, mean pulmonary artery pressure; $\mathrm{mPCWP}$, mean pulmonary capillary wedge pressure; PVR, pulmonary vascular resistance; RHC, right heart catheterization 
was downloaded with the GEO accession number GSE58095 [26].

\section{Results}

\section{Pathway analysis of protein expression}

SOMAScan protein expression was evaluated in sera from 13 subjects with lcSSc-PAH and 16 subjects with lcSSc and no clinical evidence of PAH (Table 1). Detailed hemodynamic data are found in Additional file 1: Table S1.
Eighty-two proteins were found to be differentially regulated in lcSSc-PAH patients compared to lcSSc controls (FDR $\mathrm{q} \leq 0.1$, summarized in Additional file 2: Table S2. Of the 82 proteins that were significantly different between comparing subjects with lcSSc-PAH to lcSSc-no PAH 32 were increased and 50 decreased. Analytes meeting these criteria were analyzed by unsupervised clustering for both proteins and subjects. lcSSc-PAH and lcSSc controls clustered independently (Fig. 1). An unfiltered clustering

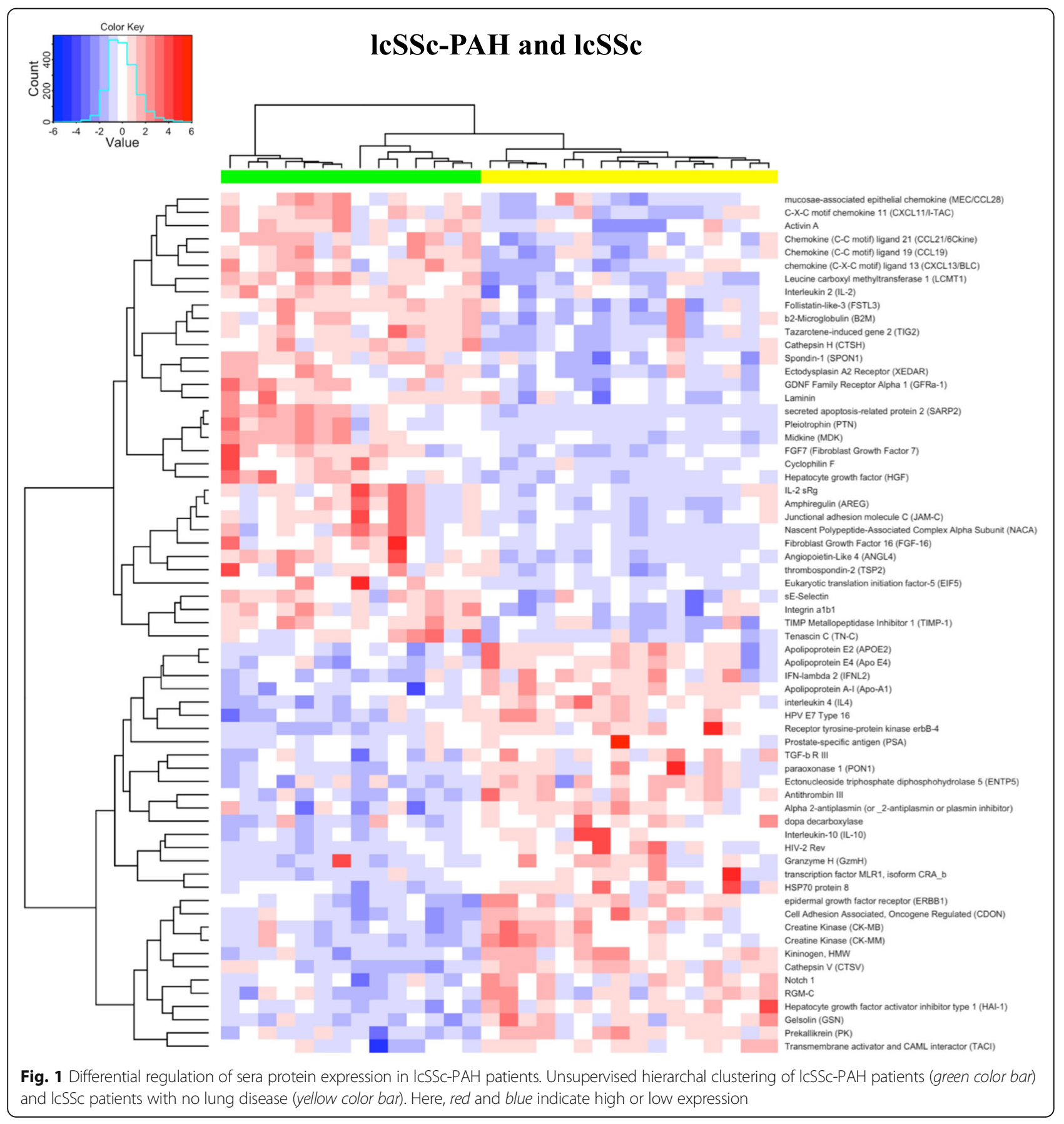


diagram of all the measured proteins was also examined. The presence of PAH was the dominant signal in the serum proteome, as all the lcSSc-PAH cases clustered together without any structured stratification of groups (Additional file 3: Figure S1).

We mined publicly available data to identify which proteins of the 82 had been previously observed as differentially regulated in skin and lung tissue or PBMCs of SSc-PAH patients (Additional file 4: Table S3). The search yielded 16 proteins with corresponding differential expression by either fold change, absolute value log2 fold-change $>1$ or adjusted $p$ value $\leq 0.1$ ( $p$ value was adjusted by Benjamini-Hochberg). About half of the identified proteins were related to the extracellular matrix including SFRP1, TNC, and TIMP1. Some immune markers were also seen as differentially regulated across data sets including complement decay-accelerating factor (CD55), CD36, CD27, and CCL19 [17, 24]. MDK was the only protein previously observed to also be differentially regulated at the mRNA level in lung tissue [25].

The 82 proteins that were differentially regulated between lcSSc-PAH and lcSSc-no PAH controls were explored in greater depth by pathway analysis. There was a cluster of chemokine signaling molecules activated including CCL19, CXCL13, CXCL11, CCL28, and CCL21, as well as several transforming growth factor beta (TGF- $\beta$ ) regulated molecules including FSTL3 and Spondin-1. Using IPA several activated upstream regulators were identified within the data, including a strong interferon gamma (IFN- $\gamma$ ) signature (Fig. 2).

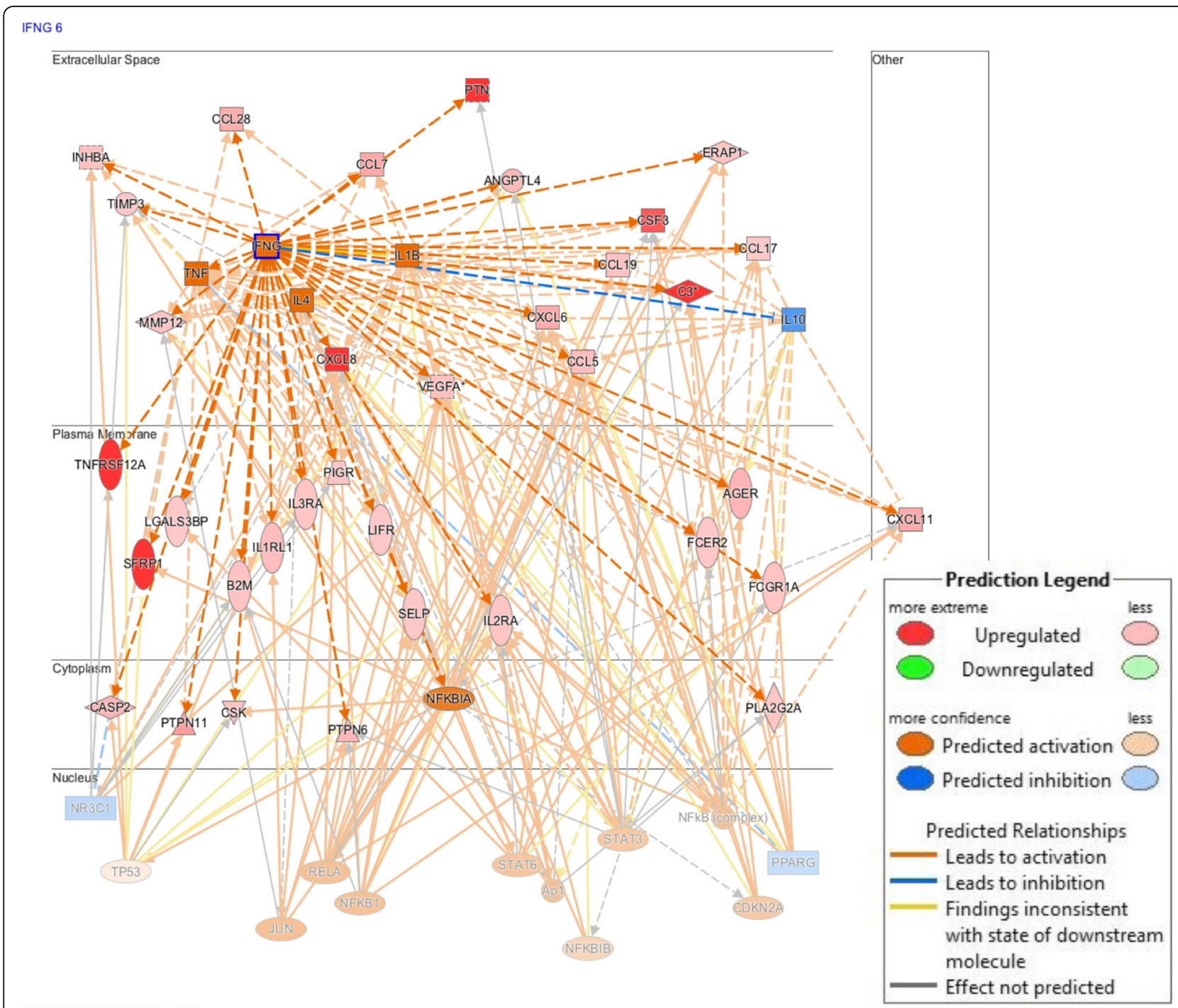

Fig. 2 INF- $\gamma$ pathway signature in sera of IcSSc-PAH patients. Graphical representation of the proteins involved the activated INF- $\gamma$ signature pathway of ICSSc-PAH patients. Colors indicate predicted activation (orange: activated, and blue: inhibited) and regulation status (red upregulated, green downregulated) of molecules in the data set. Lines between molecules depict predicted relationships based on what is known in the current literature 


\section{Putative biomarker selection and validation}

Five of the proteins upregulated in $1 \mathrm{cSS} c-\mathrm{PAH}$ sera (FSTL3, Spondin-1, junctional adhesion molecule $\mathrm{C}$ (JAM-C), CCL28, and MDK) were investigated further for their individual ability to discriminate between subjects with lcSSc-PAH and lcSSc-no PAH in silico (logistic regression) based on biological relevance, their presence in different clusters and average fold change $>2$ (Additional file 5: Figure S2). FSTL3 and MDK had nearly equal areas under the curve (AUC: 0.90 and 0.89). However, FSTL3 performed better then MDK when high sensitivity was required, and MDK performed better then FSTL3 when high specificity was required. Additionally, MDK and FSTL3 did not correlate with each other or with BNP (Additional file 6: Figure S3). This suggested that in combination these proteins would give additional information to the currently measured BNP. These proteins were investigated in combination using a generalized linear model. MDK and FSTL3 were also examined in other clinical subgroups of systemic sclerosis and were shown to be specifically upregulated compared to dcSSc, lcSSc-ILD (diagnosed by HRCT) (Fig. 3), healthy controls were included for reference.

The ability to discriminate between lcSSc subjects with and without PAH using Midkine and FSTL3 was confirmed by enzyme-linked immunosorbent assay (ELISA) on the patients originally analyzed by SOMAscan. Both proteins were elevated in subjects with SSc-PAH compared to those without PAH, and when modeled together using multiple logistic regression showed an increase in discriminatory power (Additional file 7: Figure S4).

Sera from two validation cohorts were then used to test the discrimination of Midkine and FSTL3 for lcSSc-PAH. Clinical characteristics in the validation cohorts were similar but not identical to the test cohort (Table 1, Additional file 1: Table S1 and Additional file 7: Table S4). In the validation cohorts not all samples were collected on the day of diagnostic RHC; as such, some patients were being treated for PAH. Additionally, some patients had other pulmonary complications, such as ILD. Yet, both cohorts demonstrated a consistent and significant increase $(p<0.01)$ in both FSTL3 and MDK protein concentrations in SSc-PAH patients compared to lcSSc-no PAH (Fig. 4). Logistic and multiple logistic models were created based on protein concentrations (Fig. 5). In the discovery cohort, the biomarker had an area under the ROC curve (AUC) of 0.94 (95\% confidence interval (CI) 81 to 95 ), a sensitivity of $99 \%$, and a specificity of $99 \%$. In the first validation cohort, the biomarker had an AUC of $85 \%$ (95\% CI 67 to 94), a sensitivity of $76 \%$, and a specificity of $76 \%$. In the second validation cohort, the biomarker had an AUC of 92\% (95\% CI 77 to 95), a sensitivity of $91 \%$, and a specificity of $80 \%$.

The discovery cohort was comprised of all untreated patients and one subject with ILD, while the second validation cohort was comprised of all treated patients with no subjects with ILD (Table 1, Additional file 1: Table S1, and Additional file 8: Table S4). However, the

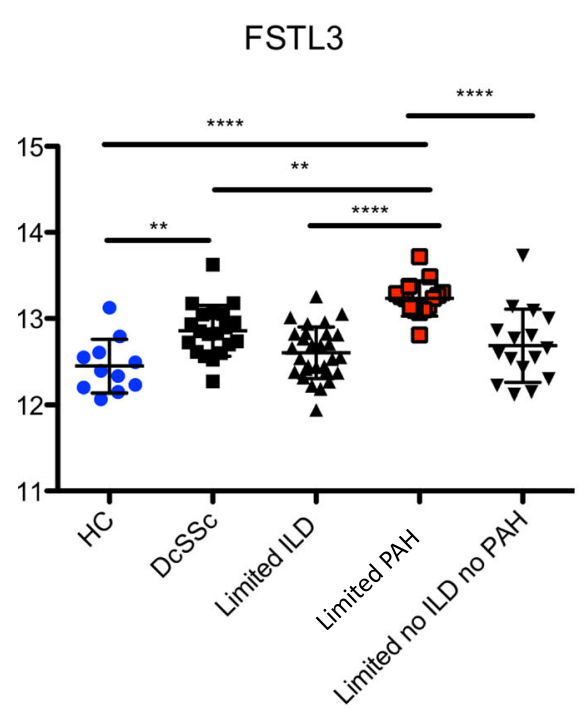

FSTL3: Follistatin-like 3

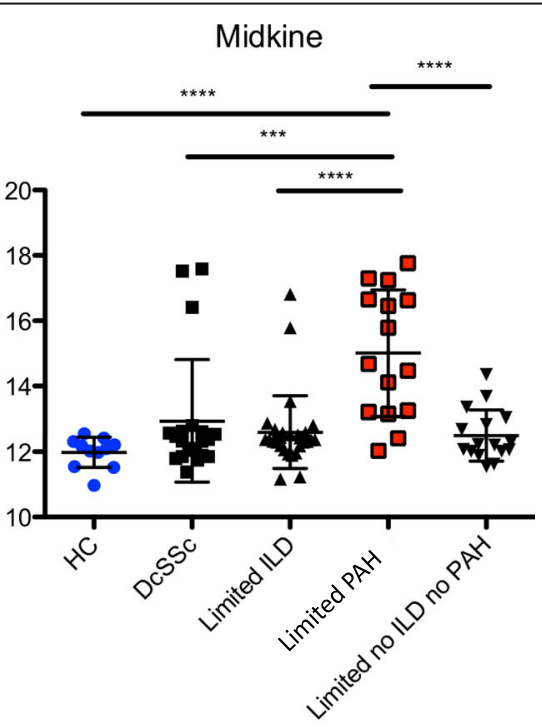

MDK(NEGF2): Midkine

Fig. 3 Midkine (MDK) and follistatin-like 3 (FSTL3) are upregulated specifically in the IcSSc-PAH population. Graphs show the differential expression of MDK and FSTL3 relative to healthy controls with a side-by-side comparison to patients with dcSSc, SSc-ILD. Data is displayed as log2 expression levels and comparisons made by ANOVA: corrected with Bonferroni's multiple comparison test. dcSsc diffuse systemic sclerosis HC healthy control, ILD interstitial lung disease, ICSSC-PAH limited cutaneous systemic sclerosis pulmonary arterial hypertension 

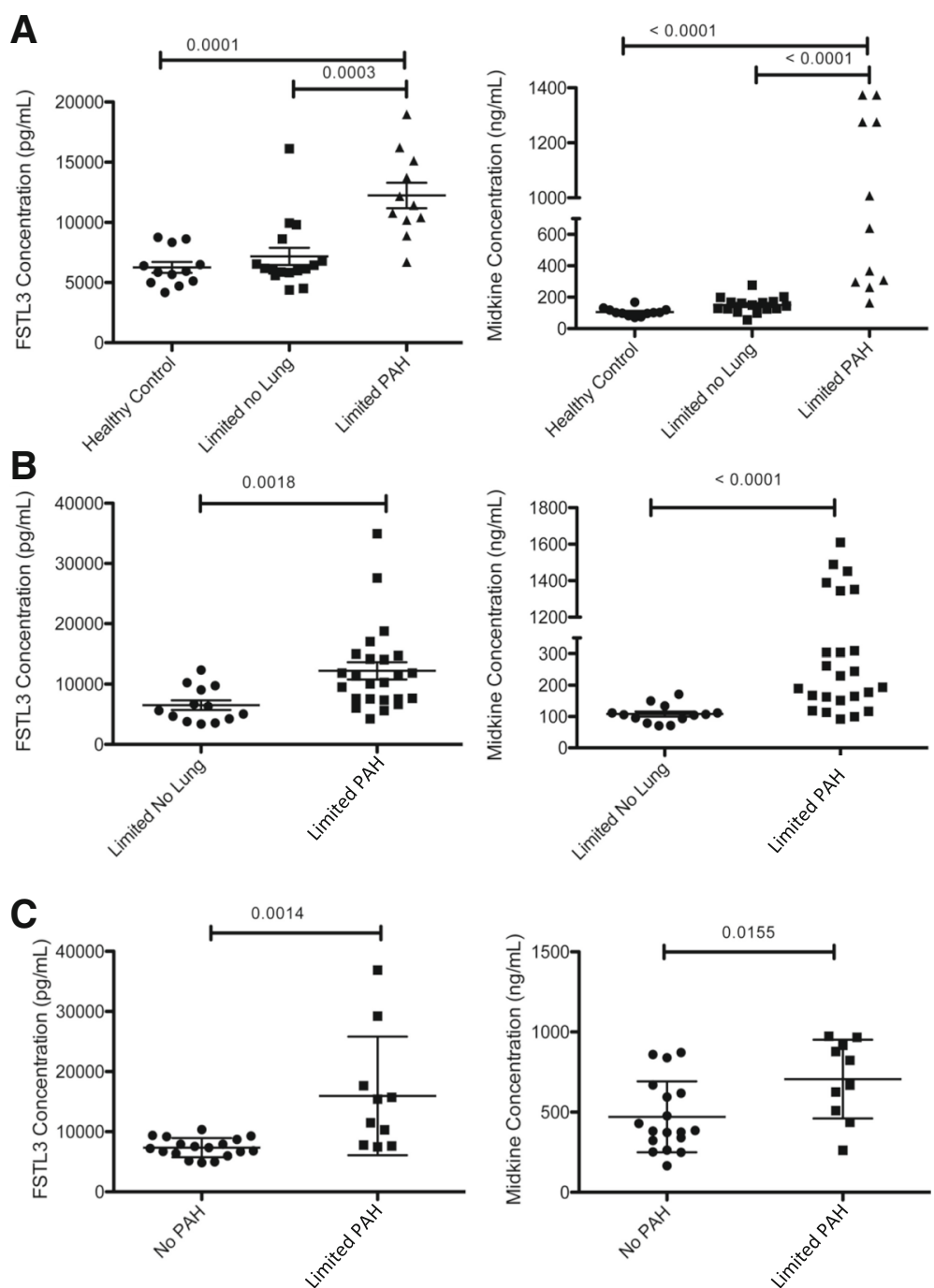

Fig. 4 ELISA validation cohort data. Graphs show differences (Wilcoxon signed-rank test) of ICSSc-PAH Follistatin-like 3 (FSTL3), and Midkine (MDK) protein concentrations as compared to SSC patients with no lung disease in the test cohort (A) as well as two independent validation cohorts (B and C). PAH pulmonary arterial hypertension

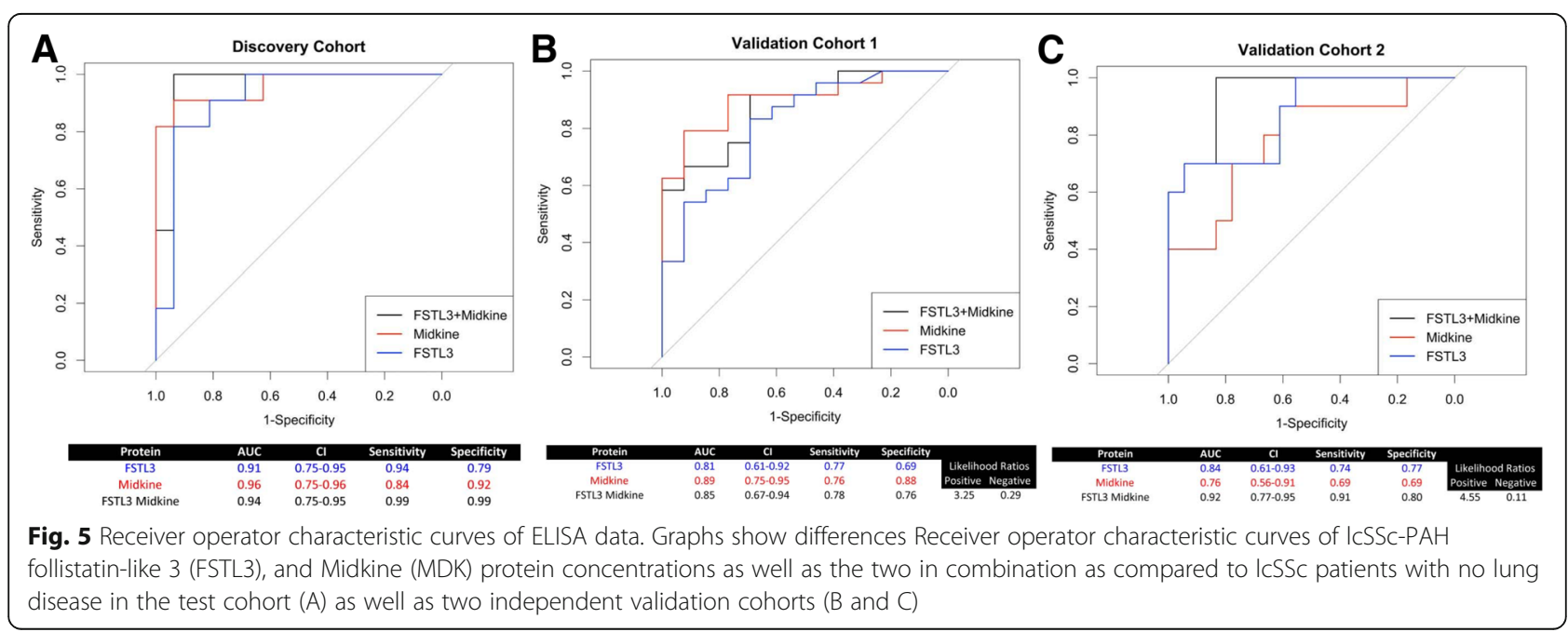


first validation cohort had a mix of treated $(n=11)$ and untreated $(n=12)$ subjects as well as a mix of subjects with $(n=3)$ and without $(n=20)$ ILD (Table 1, Additional file 8: Table S4). After excluding subjects with ILD and patients treated for PAH, FSTL3 and MDK remained elevated $(p<0.05)$ in SSc-PAH patients compared to lcSSc-no PAH (Additional file 9: Figure S5). However, exclusion of these patients did not increase the parameters of the ROCs, with the AUC including all the patients remaining the highest at 85\% (Additional file 9: Figure S5).

\section{Discussion}

Investigation into the circulating proteome of lcSSc-PAH allowed for the identification of multiple putative biomarkers. Our results show a circulating serum protein pattern that is associated with lcSSc-PAH compared to lcSSc-no $\mathrm{PAH}$, thus identifying a potential serum proteomic biomarker for use in the diagnostic evaluation of lcSSc-PAH. In sum, we demonstrated that the combination of FSTL3 and MDK are highly associated with lcSSc-PAH.

Our results show that 82 circulating serum proteins are associated with lcSSc-PAH compared to lcSSc-no $\mathrm{PAH}$; this data set allowed us a unique opportunity to examine potential upstream regulators of these proteins. Within those 82 differentially regulated proteins, there were a number of activated chemokines, CCL19, CXCL13, and CCL21, which are implicated in tertiary lymphoid structures [27]. In addition, many of the upregulated proteins are regulated by INF- $\gamma$, thus confirming previously published gene-expression patterns that observed INF regulation in PBMCs of SSc-PAH [19].

From these 82 differentially regulated proteins, we showed that the combination of FSTL3 and MDK are highly associated for SSc-PAH in the independent cohorts, with an average sensitivity of $84 \%$ and an average specificity of $79 \%$. These findings are plausible pathophysiologically. FSTL3, a TGF- $\beta$ regulated protein, inhibits the protective actions of activin A on cardiac myocytes. We also previously observed that FSTL3 is elevated, compared to healthy controls, in the sera, PBMCs, and skin of patients with dcSSc [28]. The growth factor, MDK, has been implicated in the pathogenesis of hypertension, kidney disease, and lung fibrosis [29-31].

The lack of effect of various treatments in the validation data sets on biomarker performance suggests that this is a robust diagnostic biomarker. Also, that the roles of these biomarkers are upstream from the pathophysiologic processes affected by vasodilators, the most commonly available therapeutics. Additionally, some patients had radiographic evidence of ILD as well as SSc-PAH. The effect of ILD and PAH treatment was tested in a small subset of patients in validation cohort one. Including patients with ILD also bestowed no additional benefit to the diagnostic capabilities of FSTL3 and MDK.
Some samples were not collected at the time of diagnostic RHC (Table 1 and Additional file 9: Table S4) and thus the relationship between biomarker and clinical disease is less certain in these cases. In spite of this, there was still a significant increase in MDK and FSTL3 among patients with SSc-PAH. Of particular interest are those patients whose sera was collected prior to $\mathrm{PAH}$ diagnosis suggesting that these biomarkers might be elevated before $\mathrm{PAH}$ is clinically suspected. There has not been investigation of MDK or FSTL3 in other subset of SSc patients who might have increased concentrations of those specific proteins, such as patients with cardiac involvement or renal disease.

\section{Conclusions}

In sum, we have identified serum proteins that are strongly associated with SSc-PAH. Further investigation of these and other proteins has the potential to improve the diagnosis, care, and outcomes in this clinically challenging PAH subset.

\section{Additional files}

Additional file 1: Table S1. Detailed hemodynamic measurements and clinical characteristics of the discovery cohort. (PDF $127 \mathrm{~kb}$ )

Additional file 2: Table S2. Complete list of differentially regulated proteins: 82 proteins were found to be differentially regulated in ICSSc-PAH patients compared to IcSSc controls (false discovery rate $q \leq 0.1)$. (PDF $1235 \mathrm{~kb}$ )

Additional file 3: Figure S1. Unfiltered clustering diagram of all 1129 measured proteins: Unsupervised hierarchal clustering of ICSSC-PAH patients (green color bar) and IcSSc patients with no lung disease (yellow color bar). Here, red and blue indicate high or low expression. (PDF $1774 \mathrm{~kb}$ )

Additional file 4: Table S3. Shortlist proteins previously observed as differentially regulated in skin and lung tissue or PBMCs of SSc-PAH patients. (PDF $71 \mathrm{~kb}$ )

Additional file 5: Figure S2. Receiver operator characteristic (ROC) curves of SOMAlogic: ROCs based on in silico (logistic regression). (PDF 678 kb)

Additional file 6: Figure S3. Correlations of Midkine (MDK), follistatinlike 3 (FSTL3), and BNP: protein levels of MDK, FSTL3, and BNP are plotted as linear regressions Spearman's correlation coefficients are reported in the upper left corner of each graphic. (A) Examines the correlation between FSTL3 and MDK, (B) the correlation between FSTL3 and BNP, (C) MDK and BNP. (PDF $361 \mathrm{~kb}$ )

Additional file 7: Figure S4. Concentrations of Midkine (MDK), follistatin-like 3 (FSTL3): concentrations of FSTL3 (A) and Midkine (B) were measured by ELISA and modeled together using multiple logistic regression (C). (PDF $779 \mathrm{~kb}$ )

Additional file 8: Table S4. Detailed hemodynamic measurements and clinical characteristics of validation cohorts: Table A contains the details from validation cohort one while Table $B$ contains the details from validation cohort two. (PDF 334 kb)

Additional file 9: Figure S5. Concentrations of Midkine (MDK), follistatin-like 3 (FSTL3): concentrations of FSTL3 and MDK were examined with treated subjects excluded from the analysis (A) or subjects with ILD (B). ROC parameters were examined with the exclusion of the different subsets (C). (PDF $970 \mathrm{~kb}$ ) 


\section{Abbreviations}

AUC: Area under the receiver operating characteristic curve; BNP: Brain natriuretic peptide; dcSSc: Diffuse systemic sclerosis; FDR: False discovery rate; FSTL3: Follistatin-like 3; FVC: Forced vital capacity; HRCT: High-resolution CT scan; ILD: Interstitial lung disease; INF- $\gamma$ : Interferon gamma; ICSSc: Limited cutaneous systemic sclerosis; MDK: Midkine; mPAP: Mean pulmonary artery pressure; NT-proBNP: N-terminal fragment of pro-BNP; PBMC: Peripheral blood mononuclear cell; RHC: Right heart catheterization; ROC: Receiver operating characteristic curve; SPAP: Systolic PAP; SSc: Systemic sclerosis; SSC-PAH: Systemic sclerosis-associated pulmonary arterial hypertension; TNF- $\beta$ : Transforming growth factor beta

\section{Funding}

This work was supported by National Institutes of Health, National Institute of Arthritis Musculoskeletal and Skin Disease grants; Scleroderma Core Centers (5P30AR061271), Scleroderma Center of Research Translation (1P50AR060780) and 2R01AR051089 to RL.

\section{Availability of data and materials}

The data sets generated during and analyzed during the current study are available in the supplementary information files as well as from the corresponding author on reasonable request.

\section{Authors' contributions}

LMR and JCM contributed to interpretation, design, analysis, and acquisition. $\mathrm{RL}$ and HF contributed to conception, interpretation, and acquisition. ES, RW, $\mathrm{KR}, \mathrm{NH}, \mathrm{RS}$, and RD contributed to acquisition. All authors participated in writing, reading, and approving the final manuscript.

\section{Ethics approval and consent to participate}

All subjects that consented to give tissue and serum samples were enrolled with signed informed written consent by the institutional review board of Boston University.

\section{Consent for publication}

The manuscript does not contain any individual person's data in any form.

\section{Competing interests}

The authors declare that they have no competing interests.

\section{Publisher's Note}

Springer Nature remains neutral with regard to jurisdictional claims in published maps and institutional affiliations.

\section{Author details}

${ }^{1}$ Boston University School of Medicine, E5 Arthritis Center, 72 E Concord Street, Boston, MA 0211, USA. ${ }^{2}$ Tufts University, Boston, MA, USA. ${ }^{3}$ University of Pittsburgh Medical Center, Pittsburgh, PA, USA.

\section{Received: 1 March 2018 Accepted: 18 July 2018}

Published online: 16 August 2018

\section{References}

1. Chaisson NF, Hassoun PM. Systemic sclerosis-associated pulmonary arterial hypertension. Chest. 2013;144(4):1346-56. https://doi.org/10.1378/chest.12-2396. published Online First: Epub Date

2. Chung L, Domsic RT, Lingala B, et al. Survival and predictors of mortality in systemic sclerosis-associated pulmonary arterial hypertension: outcomes from the pulmonary hypertension assessment and recognition of outcomes in scleroderma registry. Arthritis Care Res (Hoboken). 66(3):489-95. https://doi.org/10.1002/acr.22121. published Online First: Epub Date

3. Foris $\mathrm{V}$, Kovacs $\mathrm{G}$, Tscherner $\mathrm{M}$, et al. Biomarkers in pulmonary hypertension: what do we know? Chest. 2013;144(1):274-83. https://doi.org/10.1378/chest. 12-1246. published Online First: Epub Date

4. Hao Y, Thakkar V, Stevens W, et al. A comparison of the predictive accuracy of three screening models for pulmonary arterial hypertension in systemic sclerosis. Arthritis Res. Ther. 2015;17:7. https://doi.org/10.1186/s13075-0150517-5. published Online First: Epub Date

5. Proudman SM, Stevens WM, Sahhar J, et al. Pulmonary arterial hypertension in systemic sclerosis: the need for early detection and treatment. Intern.
Med. J. 2007;37(7):485-94. https://doi.org/10.1111/j.1445-5994.2007.01370.x. published Online First: Epub Date

6. Task Force for D, Treatment of Pulmonary Hypertension of European Society of C, European Respiratory S, et al. Guidelines for the diagnosis and treatment of pulmonary hypertension. Eur. Respir. J. 2009;34(6):1219-63. https:/doi.org/10. 1183/09031936.00139009. published Online First: Epub Date

7. Denton CP, Cailes JB, Phillips GD, et al. Comparison of Doppler echocardiography and right heart catheterization to assess pulmonary hypertension in systemic sclerosis. Br J Rheumatol. 1997;36(2):239-43.

8. Fisher MR, Forfia PR, Chamera E, et al. Accuracy of Doppler echocardiography in the hemodynamic assessment of pulmonary hypertension. Am. J. Respir. Crit. Care Med. 2009;179(7):615-21. https://doi. org/10.1164/rccm.200811-16910C. published Online First: Epub Date

9. Badesch DB, Champion HC, Sanchez MA, et al. Diagnosis and assessment of pulmonary arterial hypertension. J. Am. Coll. Cardiol. 2009;54(1 Suppl):S55-66. https://doi.org/10.1016/j.jacc.2009.04.011. published Online First: Epub Date

10. McGoon M, Gutterman D, Steen V, et al. Screening, early detection, and diagnosis of pulmonary arterial hypertension: ACCP evidence-based clinical practice guidelines. Chest. 2004;126(1 Suppl):14S-34S. https://doi.org/10. 1378/chest.126.1_suppl.14S. published Online First: Epub Date

11. Cavagna L, Caporali R, Klersy C, et al. Comparison of brain natriuretic peptide (BNP) and NT-proBNP in screening for pulmonary arterial hypertension in patients with systemic sclerosis. J. Rheumatol. 2010;37(10):2064-70. https://doi. org/10.3899/jrheum.090997. published Online First: Epub Date

12. Mathai SC, Bueso M, Hummers LK, et al. Disproportionate elevation of Nterminal pro-brain natriuretic peptide in scleroderma-related pulmonary hypertension. Eur Respir J. 2010;35(1):95-104. https://doi.org/10.1183/ 09031936.00074309. published Online First: Epub Date

13. Williams MH, Das C, Handler CE, et al. Systemic sclerosis associated pulmonary hypertension: improved survival in the current era. Heart. 2006; 92(7):926-32. https://doi.org/10.1136/hrt.2005.069484. published Online First: Epub Date

14. Lorenzen JM, Nickel N, Kramer R, et al. Osteopontin in patients with idiopathic pulmonary hypertension. Chest. 2011;139(5):1010-7. https://doi. org/10.1378/chest.10-1146. published Online First: Epub Date

15. Malhotra R, Paskin-Flerlage S, Zamanian RT, et al. Circulating angiogenic modulatory factors predict survival and functional class in pulmonary arterial hypertension. Pulm Circ. 2013;3(2):369-80. https://doi.org/10.4103/ 2045-8932.110445. published Online First: Epub Date

16. Tamura $Y$, Ono T, Kuwana M, et al. Human pentraxin 3 (PTX3) as a novel biomarker for the diagnosis of pulmonary arterial hypertension. PLoS One. 2012;7(9):e45834. https://doi.org/10.1371/journal.pone.0045834. published Online First: Epub Date

17. Pendergrass SA, Hayes E, Farina G, et al. Limited systemic sclerosis patients with pulmonary arterial hypertension show biomarkers of inflammation and vascular injury. PLoS One. 2010;5(8):e12106. https://doi.org/10.1371/journal. pone.0012106. published Online First: Epub Date

18. Golub TR, Slonim DK, Tamayo P, et al. Molecular classification of cancer: class discovery and class prediction by gene expression monitoring. Science. 1999;286(5439):531-7.

19. Christmann RB, Hayes $E$, Pendergrass $S$, et al. Interferon and alternative activation of monocyte/macrophages in systemic sclerosis-associated pulmonary arterial hypertension. Arthritis Rheum. 2011:63(6):1718-28. https://doi.org/10.1002/art.30318. published Online First: Epub Date

20. Simonneau G, Gatzoulis MA, Adatia I, et al. Updated clinical classification of pulmonary hypertension. J. Am. Coll. Cardiol. 2013;62(25 Suppl):D34-41. https://doi.org/10.1016/j.jacc.2013.10.029. published Online First: Epub Date

21. Grund B, Sabin C. Analysis of biomarker data: logs, odds ratios, and receiver operating characteristic curves. Curr Opin HIV AIDS. 2010;5(6):473-9. https:// doi.org/10.1097/COH.0b013e32833ed742. published Online First: Epub Date

22. Schisterman EF, Perkins NJ, Liu A, et al. Optimal cut-point and its corresponding Youden index to discriminate individuals using pooled blood samples. Epidemiology. 2005;16(1):73-81.

23. Cheadle C, Berger AE, Mathai SC, et al. Erythroid-specific transcriptional changes in PBMCs from pulmonary hypertension patients. PLoS One. 2012; 7(4):e34951. https://doi.org/10.1371/journal.pone.0034951. published Online First: Epub Date

24. Risbano MG, Meadows CA, Coldren CD, et al. Altered immune phenotype in peripheral blood cells of patients with scleroderma-associated pulmonary hypertension. Clin. Transl. Sci. 2010;3(5):210-8. https://doi.org/10.1111/j.17528062.2010.00218.x. published Online First: Epub Date 
25. Hsu ESH, Jordan RM, Lyons-Weiler J, Pilewski JM, Feghali-Bostwick CA. Lung tissues in patients with systemic sclerosis have gene expression patterns unique to pulmonary fibrosis and pulmonary hypertension. Arthritis Rheum. 2011:63(3):783-94.

26. Assassi S, Swindell WR, Wu M, et al. Dissecting the heterogeneity of skin gene expression patterns in systemic sclerosis. Arthritis Rheumatol. 2015; 67(11):3016-26. https://doi.org/10.1002/art.39289. published Online First: Epub Date

27. Ruddle NH. Lymphatic vessels and tertiary lymphoid organs. J. Clin. Invest. 2014;124(3):953-9. https://doi.org/10.1172/JCl71611. published Online First: Epub Date

28. Rice LM, Mantero JC, Stifano G, et al. A Proteome-Derived Longitudinal Pharmacodynamic Biomarker for Diffuse Systemic Sclerosis Skin. J. Invest. Dermatol. 2017;137(1):62-70. https://doi.org/10.1016/j.jid.2016.08.027. published Online First: Epub Date

29. Cohen S, Shachar I. Midkine as a regulator of B cell survival in health and disease. Br. J. Pharmacol. 2014;171(4):888-95. https://doi.org/10.1111/bph. 12419. published Online First: Epub Date

30. Sato W, Sato Y. Midkine in nephrogenesis, hypertension and kidney diseases. Br. J. Pharmacol. 2014;171(4):879-87. https://doi.org/10.1111/bph. 12418. published Online First: Epub Date

31. Zhang R, Pan Y, Fanelli V, et al. Mechanical Stress and the Induction of Lung Fibrosis via the Midkine Signaling Pathway. Am. J. Respir. Crit. Care Med. 2015;192(3):315-23. https://doi.org/10.1164/rccm.201412-2326OC. published Online First: Epub Date

Ready to submit your research? Choose BMC and benefit from:

- fast, convenient online submission

- thorough peer review by experienced researchers in your field

- rapid publication on acceptance

- support for research data, including large and complex data types

- gold Open Access which fosters wider collaboration and increased citations

- maximum visibility for your research: over $100 \mathrm{M}$ website views per year

At $\mathrm{BMC}$, research is always in progress.

Learn more biomedcentral.com/submissions 